\title{
Correction to: Hepatic Capillaria hepatica (Bancroft, 1893) infection in cat (Felis catus) - histopathological findings and first report from Iran
}

\author{
Alireza Sazmand $^{1,2}$ (D) $\cdot$ Monireh Khordadmehr ${ }^{3}$ (D) Alireza Nourian $^{1}$ (D) $\cdot$ Domenico Otranto $^{1,4}$ (D) \\ Published online: 22 February 2021 \\ (C) Springer-Verlag GmbH Germany, part of Springer Nature 2021
}

\section{Correction to: Parasitol Res}

https://doi.org/10.1007/s00436-021-07056-4

Affiliation of Monireh Khordadmehr was incorrectly assigned as 2 in the original version of this article when in fact it should have been 3. Also affiliation of Domenico Otranto was incorrectly assigned as 1,3,4 in the original version of this article when in fact it should have been 1,4. Correct affiliations are presented here.

The original article has been corrected.

Publisher's note Springer Nature remains neutral with regard to jurisdictional claims in published maps and institutional affiliations.

The online version of the original article can be found at https://doi.org/ $10.1007 / \mathrm{s} 00436-021-07056-4$

Alireza Nourian

nourian@basu.ac.ir

Alireza Sazmand

alireza.sazmand@basu.ac.ir

Monireh Khordadmehr

khordadmehr@tabrizu.ac.ir

Domenico Otranto

domenico.otranto@uniba.it

1 Department of Pathobiology, Faculty of Veterinary Science, Bu-Ali Sina University, Hamedan 6517658978, Iran

2 Zoonotic Diseases Research Center, School of Public Health, Shahid Sadoughi University of Medical Sciences, Yazd 8915173160, Iran

3 Department of Pathobiology, Faculty of Veterinary Medicine, University of Tabriz, Tabriz, Iran

4 Department of Veterinary Medicine, University of Bari, 70010 Bari, Italy 